\title{
ON THE AUTOMORPHISM GROUPS OF A COMPACT BORDERED RIEMANN SURFACE OF GENUS FIVE
}

\author{
By Kenji NaKagawa
}

\section{$\S 1$. Introduction.}

Let $S$ be a compact bordered Riemann surface of genus $g$ with $k$ boundary components. If $2 g+k-1 \geqq 2$, the automorphism group of $S$ is a finite group. Then, we put $N(g, k)$ the maximum order of automorphism groups of $S$ where the maximum is taken over all $S$ of genus $g$ with $k$ boundary components. It is well known that $N(g, k)$ is equal to the maximum order of automorphism groups of compact Riemann surfaces of genus $g$ deleted $k$ points, and every automorphism group of $S$ is isomorphic to that of a compact Riemann surface (Oikawa [7]). For every $k \geqq 0, N(0, k), N(1, k), N(2, k), N(3, k)$ and $N(4, k)$ are determined by Heins [2], Oikawa [7], Tsuji [8], Tsuji [9] and Kato [4], respectively. In the present paper, we shall determine $N(5, k)$.

Theorem. $N(5, k)$ is

(1) 192 for $k \equiv 0,24,64,88(\bmod 96)$,

(2) 160 for $k \equiv 0,32(\bmod 40)$ except the case $(1)$,

(3) 120 for $k \equiv 0,12,40,52(\bmod 60)$ except the cases (1), (2),

(4) 96 for $k \equiv 16,32,40,48,56,72(\bmod 96)$ except the cases $(2)$, (3),

(5) 80 for $k \equiv 16(\bmod 40)$ except the cases $(1),(2),(4)$,

(6) 64 for $k \equiv 0(\bmod 8)$ except the cases $(1) \sim(5)$,

(7) 60 for $k \equiv 20,32(\bmod 60)$ except the cases $(1),(2),(4) \sim(6)$,

(8) 48 for $k \equiv 0,4(\bmod 12)$ except the cases $(1) \sim(7)$,

(9) 40 for $k \equiv 0,2(\bmod 10)$ except the cases $(1) \sim(8)$,

(10) 32 for $k \equiv 4(\bmod 16)$ except the cases $(1) \sim(5),(7) \sim(9)$,

(11) 30 for $k \equiv 0,2,5,7(\bmod 15)$ except the cases $(1) \sim(10)$,

(12) 24 for $k \equiv 2,6,10,14,20(\bmod 24)$ except the cases $(1) \sim(5)$, (7), (9) $\sim(11)$,

(13) 22 for $k \equiv 0,1,2,3(\bmod 11)$ except the cases $(1) \sim(12)$,

(14) 20 for $k \equiv 1,5,7,11(\bmod 20)$ except the cases $(1) \sim(8),(10) \sim(13)$,

(15) 16 for $k \equiv 2,6(\bmod 16)$ except the cases $(1) \sim(5),(7) \sim(9),(11) \sim(14)$,

(16) 15 for $k \equiv 1,6(\bmod 15)$ except the cases $(1) \sim(10),(12) \sim(15)$,

(17) 12 for $k \equiv 0,1,3,4(\bmod 6)$ except the cases $(1) \sim(5),(7),(9) \sim(12)$,

(18) 8 otherwise.

Received September 28, 1985 
The author wishes to express his thanks to Professor N. Suita and Professor $T$. Kato for their many advices and encouragement.

\section{§2. Notation.}

Let $S$ be a compact Riemann surface of genus $g \geqq 2$, let $G$ be a conformal automorphism group of $S$ and let $N$ be the order of $G$. Let $S_{0}=S / G$ be the quotient surface with conformal structure induced from $S$ through $\pi$, where $\pi$ is the projection mapping of $S$ onto $S_{0}$. Let $g_{0}$ be the genus of $S_{0}$. At $p \in S$ and at $p_{0}=\pi(p) \in S_{0}$, by a suitable choice of local parameters, $\pi$ is represented locally by $z_{0}=z^{\nu}$, where $\nu$ is a positive integer, $z, z_{0}$ are the local parameters at $p, p_{0}$, respectively. If $\nu>1, p$ is called a branch point of multiplicity $\nu$. If $\pi\left(p_{1}\right)=\pi\left(p_{2}\right)\left(p_{1}, p_{2} \in S\right)$, then the multiplicity of $p_{1}$ is equal to that of $p_{2}$. Therefore we can define the multiplicity over $p_{0} \in S$ by the multiplicity of $p \in \pi^{-1}\left(p_{0}\right)$. Let $\left\{q_{1}, \cdots, q_{t}\right\}$ be the set of points on $S_{0}$ which are the projection of all the branch points on $S$. Let $\nu_{1}, \cdots, \nu_{t}$ be the multiplicities over $q_{1}, \cdots, q_{t}$, respectively. We call the set of integers $g_{0}, \nu_{1}, \cdots, \nu_{t}$ the signature of $G$ and denote it by $\left(g_{0} ; \nu_{1}, \cdots, \nu_{t}\right)$. Without loss of generality, we may assume $\nu_{1} \leqq \nu_{2} \leqq \cdots \leqq \nu_{t}$. For simplicity's sake, we shall denote $\left(0 ; \nu_{1}, \cdots, \nu_{t}\right)$ by $\left(\nu_{1}, \cdots, \nu_{t}\right)$.

\section{§3. Lemmas.}

LEMMA 1. (the Riemann-Hurwitz relation)

$$
2 g-2=N\left(2 g_{0}-2\right)+N \sum_{j=1}^{t}\left(1-1 / \nu_{j}\right) .
$$

LEMmA 2. (Harvey [1]) There exist a compact Riemann surface $S$ and a cyclic automorphism group $Z_{N}$ on $S$ of order $N$ with signature $\left(g_{0} ; \nu_{1}, \cdots, \nu_{t}\right)$ if and only if this signature satisfies the following l.c.m. condition (1) (4), where $M=$ l.c.m. $\left(\nu_{1}, \cdots, \nu_{t}\right)$ : the least common multiple of $\nu_{1}, \cdots, \nu_{t}$.

(1) $M=$ l.c.m. $\left(\nu_{1}, \cdots, \check{\nu}_{\jmath}, \cdots, \nu_{t}\right) .(j=1, \cdots, t)$

Here, $\check{\nu}_{j}$ denetes the omission of $\nu_{j}$.

(2) $M \mid N$ and if $g_{0}=0$, then $M=N$.

(3) $t \neq 1$ and if $g_{0}=0$, then $t \geqq 3$.

(4) If $2 \mid M$, the number of $\nu_{j}$ 's which are divisible by the maximum power of 2 that divides $M$ is even.

LEMMA 3. If $S$ has an automorphism group of order $N$ with signature $\left(g_{0} ; \nu_{1}, \cdots, \nu_{t}\right)$, then for $k=m N+\sum_{j=1}^{t} \varepsilon_{j} N / \nu_{j}, N(g, k) \geqq N$, where $m$ is a nonnegative integer and $\varepsilon_{j}=0$ or $1(\jmath=1, \cdots, t)$. 
By Lemma 3, $N(g, k)$ is completely determined by the signature of automorphism groups rather than by automorphism groups themselves. We do not look for the group of maximum order for a given integer $k$, or rather, we look for $k$ points to be deleted from a compact Riemann surface so that these $k$ points are invariant by the automorphism group with given signature.

LEMMA 4. If $p \in S$ is a fixed point of some non-trivial automorphism in $G$, then the stabilizer subgroup of $p$ in $G$ is a cyclic group. Then, the order $N$ of $G$ must be a multiple of the order of the stabilizer subgroup of $p$.

Proof. An automorphism $h$ which fixes $p$ is expanded locally as

$$
h(z)=a z+b z^{2}+\cdots \quad(a \neq 0),
$$

by a local parameter $z$ at $p$. Here $a$ is independent on the choice of local parameter. If the order of $h$ is $\nu, a$ is a primitive $\nu$ th root of unity. We claim that if $a=1$, then $h$ is indeed the identity automorphism. Let $D=\{|w|<1\}$ be the universal covering surface of $S$ and $\phi$ be the covering projection such that $\phi(0)=p$. By the covering surface theory, there is an automorphism $H$ of $D$ such that

$$
h \circ \psi=\phi \circ H
$$

and $H$ fixes the origin $w=0$. Then $H$ is an elliptic transformation and has the expansion

$$
H(w)=w+\cdots .
$$

Then $H$ is the identity. This implies that $h$ is also the identity automorphism. Since the set of all the automorphisms $h_{\imath}$ fixing $p$ is a group, the set of all the leading coefficients $a_{\imath}$ of the expansions of those automorphisms also forms a group. This group of coefficients $\left\{a_{i}\right\}$ is a cyclic group. By the above arguement, $a_{i}=a$, implies that $h_{i}=h_{\mathrm{j}}$. Thus, we conclude that the stabilizer subgroup is a cyclic group.

LEMmA 5. (Wiman [10], Nakagawa [6]) If $\nu$ is the order of a stabilizer subgroup of $G$, then $2 \leqq \nu \leqq 4 g+2$.

LEMMA 6. There exists neither an automorphism of order 7 nor that of order 9 on any compact Riemann surface of genus 5 .

Proof. If $N=7$, by Lemma $4, \nu_{j}=7(j=1, \cdots, t)$. Then by the RiemannHurwitz relation we obtain

$$
8=14\left(g_{0}-1\right)+6 t .
$$

Since $g_{0} \geqq 0, t \geqq 0$, this equation has no integer solution. Then the automorphism of order 7 does not exist. If $N=9$, by Lemma $4, \nu_{j}=3$ or 9 . Then, by the Riemann-Hurwitz relation we obtain 


$$
8=18\left(g_{0}-1\right)+6 r+8 s \quad(r+s=t) .
$$

This equation has the integer solutions $(1 ; 9)$ and $(0 ; 3,3,3,9)$. But these two solutions do not satisfy the 1.c. m. condition.

LEMMA 7. For all $k \geqq 0, N(5, k) \geqq 8$.

Proof. Let $S$ be the Riemann surface defined by

$$
y^{8}=x^{4}(x-1)^{2}(x-\alpha)
$$

where $\alpha$ is a complex number which is not equal to 0,1 . Let $h$ be the automorphism of $S$ defined by

$$
h(x, y)=(x, \exp (\pi \imath / 4) y) .
$$

The automorphism group $\langle h\rangle$ is of order 8 with signature $(2,4,8,8)$. Since $k=8 m+4 \varepsilon_{1}+2 \varepsilon_{2}+\varepsilon_{3}+\varepsilon_{4}$ represents arbitrary integer by a suitable choice of $m$ and $\varepsilon,(\jmath=1, \cdots, 4)$, then by Lemma 3 we obtain that $N(5, k) \geqq 8$.

From now on we are going to determine whether the automorphism group with a given signature exists or not on a compact Riemann surface of genus 5. By Lemma 7, it is not necessary to consider the groups of order $\leqq 8$. We assume $N>8$. By the Riemann-Hurwitz relation we obtain $g_{0} \leqq 1, t \leqq 5$. So by Lemma 5 , it is enough to consider at most finite number of signatures. Among these signatures, say, $(2,3,7)$ does not exist, since by Lemma 6 , a cyclic group of order 7 does not exist. $(2,3,15)$ also does not exist, for the order 80 is not a multiple of 3 . In a similar way, using Lemmas 1,4 and 6 , we find that many signatures do not exist. Then, it is enough to consider the following signatures :

$\begin{array}{ccll}\text { order signature } & & \\ 192(2,3,8) & 160(2,4,5) & 120(2,3,10) & 96(2,3,12) \\ 96(2,4,6) & 96(3,3,4) & 80(2,5,5) & 66(2,3,22) \\ 64(2,4,8) & 60(2,5,6) & 60(3,3,5) & 48(2,4,12) \\ 48(2,6,6) & 48(3,3,6) & 48(3,4,4) & 40(2,4,20) \\ 40(2,5,10) & 33(3,3,11) & 32(2,8,8) & 32(4,4,4) \\ 30(2,6,15) & 30(3,3,15) & 30(3,5,5) & 24(2,12,12) \\ 24(3,4,12) & 24(3,6,6) & 24(4,4,6) & 22(2,11,22) \\ 20(2,20,20) & 20(4,4,10) & 20(5,5,5) & 16(4,8,8) \\ 15(3,15,15) & 15(5,5,15) & 12(6,12,12) & 11(11,11,11) \\ 48(2,2,2,3) & 32(2,2,2,4) & 24(2,2,2,6) & 24(2,2,3,3) \\ 20(2,2,2,10) & 16(2,2,4,4) & 12(2,2,4,12) & 12(2,2,6,6) \\ 12(2,3,3,6) & 12(2,3,4,4) & 12(3,3,3,3) & 10(2,2,10,10) \\ 16(2,2,2,2,2) & 12(2,2,2,2,3) & 10(2,2,2,2,5) & 16(1 ; 2) \\ 12(1 ; 3) & 10(1 ; 5) & & \end{array}$




\section{$\S 4$. The existence of hyperelliptic surfaces.}

LEMMA 8. Let $\alpha_{1}, \cdots, \alpha_{2 g+2}$ be distinct complex numbers and let $f$ be a linear transformation of the sphere which leaves the set $\left\{\alpha_{1}, \cdots, \alpha_{2 g+2}\right\}$ invariant. Then, there are two automorphisms $h_{1}, h_{2}$ on the hyperelliptic surface defined by

$$
y^{2}=\prod_{n=1}^{2 g+2}\left(x-\alpha_{n}\right)
$$

such that $f \circ x=x \circ h,(\jmath=1,2)$.

At first, using Lemma 8, we show the existence of the group with signature $(2,3,10)$ of order 120 . On the Riemann sphere we choose the set of 12 points $\alpha_{1}, \cdots, \alpha_{12}$ which forms the verteces of the icosahedron. The icosahedral group leaves the set of these 12 points invariant and its order is 60 . Then by Lemma 8 the hyperelliptic surface defined by

$$
y^{2}=\prod_{n=1}^{12}\left(x-\alpha_{n}\right)
$$

has the automorphism group of order 120 with signature $(2,3,10)$. Secondly, we show the existence of the group with signature $(2,4,12)$ of order 48 . We put $\alpha_{n}=\exp (\pi$ in $/ 6)(n=0,1, \cdots, 11)$. The dihedral group generated by the linear transformations

$$
x \rightarrow \exp (\pi \imath / 6) x, \quad x \rightarrow 1 / x
$$

leaves $\left\{\alpha_{n}\right\}$ invariant and its order is 24 . Thus the hyperelliptic surface defined by

$$
y^{2}=x^{12}-1
$$

has the automorphism group of order 48 with signature $(2,4,12)$. By the similar way we can show the existence of the following signatures. We shall list up the order $N$ of $G$, the signature, $\left\{\alpha_{n}\right\}$ and $G_{0}$ (the group of linear transformations of the sphere that leaves $\left\{\alpha_{n}\right\}$ invariant.)

$N$ signature
$120(2,3,10)$
$48(2,4,12)$
$40(2,4,20)$
$24(2,12,12)$
$24(4,4,6)$
$24(2,2,3,3)$
$22(2,11,22)$
$20(2,20,20)$
$20(4,4,10)$
$12(2,3,4,4)$

$$
\left\{\alpha_{n}\right\}
$$

vertices of icosahedron

$\exp (\pi$ in $/ 6) \quad(n=0,1, \cdots, 11)$

$0, \infty, \exp (\pi$ in $/ 5)(n=0,1, \cdots, 9)$

$\exp (\pi$ in $/ 6) \quad(n=0,1, \cdots, 11)$

$\exp (\pi$ in $/ 6) \quad(n=0,1, \cdots, 11)$

12 points invariant by $T$

$0, \exp (2 \pi$ in $/ 11)(n=0,1, \cdots, 10)$

$0, \infty, \exp (\pi$ in/5) $(n=0,1, \cdots, 9)$

$0, \infty, \exp (\pi$ in $/ 5)(n=0,1, \cdots, 9)$

$\exp (2 \pi$ in $/ 3) / 2, \exp (2 \pi$ in $/ 3)$,

$2 \exp (2 \pi i n / 3)(n=0,1,2)$
$G_{0}$

icosahedral group I

dihedral group $D_{12}$

dihedral group $D_{10}$

cyclic group $Z_{12}$

dihedral group $D_{6}$ tetrahedral group $T$

cyclic group $Z_{11}$

cyclic group $Z_{10}$

dihedral group $D_{5}$

dihedral group $D_{3}$ 
Finally we show the existence of the signature $(6,12,12)$. On the surface defined by

$$
y^{12}=x(x-1)
$$

let $h$ be the automorphism

$$
h(x, y)=(x, \exp (\pi i / 6) y) .
$$

Then $\langle h\rangle$ is a group with signature $(6,12,12)$. The existence of the group of order 60 with signature $(3,3,5)$ is shown later in $\S 5$.

\section{$\S 5$. The existence of non-hyperelliptic surfaces.}

According to Wiman [11], there exist the automorphism groups of orders 192, 160, 96 and 64. The signature of the group of order 192 is $(2,3,8)$. Then there are a Fuchsian triangle group $\Gamma$ with signature $(2,3,8)$ and the normal subgroup $K$ of $\Gamma$ of index 192 without elliptic elements such that $G$ is isomorphic to $\Gamma / K$. We construct the non-Euclidean triangle $A B C$ in the unit disk in $w$-plane, as follows. The angles at the vertices $A, B$ and $C$ are $\pi / 8, \pi / 2$ and $\pi / 3$, respectively. Put $A$ at the origin $w=0, B$ on the non-Euclidean half line $\{\arg w=0\}$ and $C$ on the half line $\{\arg w=\pi / 8\}$. We define $a$ by the rotation at $A$ of angle $\pi / 4, b$ by the elliptic transformation with fixed points $B$ and $B^{*}$ (the inverse point of $B$ with respect to the unit circle) of angle $\pi$ and $c$ by the elliptic transformation with fixed points $C$ and $C^{*}$ of angle $2 \pi / 3$. Then $a^{8}=b^{2}$ $=c^{3}=a b c=i d$, and $\Gamma$ is generated by $a, b$ and $c$. If we put $\bar{a}, \bar{b}$ and $\bar{c}$ the $K$ cosets of $a, b$ and $c$, respectively, then $G=\langle\bar{a}, \bar{b}\rangle . \quad a^{2}$ is the rotation at the origin of angle $\pi / 2$. Then $\left\langle a^{2}, c\right\rangle$ is a Fuchsian group whose fundamental region has the non-Euclidean area twice that of $\Gamma$. Then $\left\langle\bar{a}^{2}, \bar{c}\right\rangle$ is the automorphism group of order 96 with signature $(3,3,4) . \quad b a^{2} b$ is the elliptic transformation of angle $\pi / 2$ with fixed points $b(A)$ and $b(A)^{*}$. Then $\left\langle a, b a^{2} b\right\rangle$ is a Fuchsian group whose fundamental region has the non-Euclidean area thrice that of $\Gamma$. Then $\left\langle\bar{a}, \bar{b} \bar{a}^{2} \bar{b}\right\rangle$ is the automorphism group of order 64 with signature $(2,4,8) . \quad b a^{4} b$ is the elliptic transformation of angle $\pi$ with fixed points $b(A)$ and $b(A)^{*}$. Then $\left\langle a, b a^{4} b\right\rangle$ is the automorphism group of order 32 with signature $(2,8,8)$. $b a b a^{4} b a b$ is the elliptic transformation of angle $\pi / 4$ with fixed points $b a^{4} b(A)$ and $b a^{4} b(A)^{*}$. Then $\left\langle\bar{a}, \bar{b} \bar{a} \bar{b} \bar{a}^{4} \bar{b} \bar{a} \bar{b}\right\rangle$ is the automorphism group of order 16 with signature $(4,8,8)$. The signature of the group of order 160 is $(2,4,5)$. This group is isomorphic to $\Gamma / K$, where $\Gamma$ is a Fuchsian group with signature $(2,4,5): \Gamma=\left\langle a, b, c \mid a^{5}=b^{2}=c^{4}=a b c=i d\right\rangle$. Then $\left\langle\bar{a}, \bar{c}^{2}\right\rangle$ is the automorphism group of order 80 with signature $(2,5,5)$. Now we show the signature of the group of order 96 in Wiman's paper $[11]$ is $(2,4,6)$. We shall show later in $\S 6$, that the group of order 96 with signature $(2,3,12)$ does not exist. So the signature of the group of order 96 must be $(3,3,4)$ or $(2,4,6)$. On the curve in Wiman's paper : 


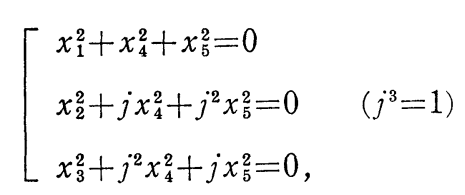

the points ${ }^{t}\left(1, j, j^{2}, 0, \pm i\right)$ are the fixed points of the linear transformation of $P^{4}$ :

$$
\left[\begin{array}{rrrrc}
0 & 0 & -1 & 0 & 0 \\
-1 & 0 & 0 & 0 & 0 \\
0 & -1 & 0 & 0 & 0 \\
0 & 0 & 0 & \jmath & 0 \\
0 & 0 & 0 & 0 & -j^{2}
\end{array}\right]
$$

of order 6. Then the automorphism group of this curve has the signature $(2,4,6)$. Finally we show the existence of $(2,6,15)$ and $(3,15,15)$. On the surface defined by

$$
y^{3}=x^{2}\left(x^{5}-1\right),
$$

put $h_{1}(x, y)=(\exp (2 \pi i / 5) x, \exp (4 \pi i / 15) y)$ and $h_{2}(x, y)=\left(1 / x,-y / x^{3}\right)$. Then $\left\langle h_{1}\right\rangle$, $\left\langle h_{1}, h_{2}\right\rangle$ are the automorphism groups with signature $(3,15,15),(2,6,15)$, respectively. Here, we show the existence of $(3,3,5)$ described in $\S 4$. The group of order 120 with signature $(2,3,10)$ is isomorphic to $\Gamma / K$, where $\Gamma$ is a Fuchsian group with signature $(2,3,10): \Gamma=\left\langle a, b, c \mid a^{10}=b^{2}=c^{3}=a b c=i d\right\rangle$. Then $\left\langle\bar{a}^{2}, \bar{c}\right\rangle$ is an automorphism group of order 60 with signature $(3,3,5)$.

\section{§ 6. The non-existence of signatures.}

The universal covering surface of $S$ is the unit disk $D=\{|w|<1\}$. Let $p \in S$ be a branch point of $\pi$ of multiplicity $\nu$, and $\psi$ the projection such that $\phi(0)=p$. A generator $h$ of the stabilizer subgroup of $p$ is lifted to the rotation

$$
w \rightarrow \exp (2 \pi i / \nu) w .
$$

Then, the Dirichlet region $F_{K}$ of $K$ centered at 0 (i. e. $F_{K}=\{w \mid d(0, w) \leqq$ $d(\tau 0, w), \tau \in K\}$, where $d($,$) denotes the non-Euclidean distance in D)$ is symmetric with respect to the rotation $w \rightarrow \exp (2 \pi i / \nu) w$. Now there is a Fuchsian group $\Gamma$ such that $G$ is isomorphic to $\Gamma / K$. So $F_{K}$ is a finite union of $F_{\Gamma}$ the Dirichlet region of $\Gamma$. The number of $F_{\Gamma}$ 's in one $F_{K}$ is equal to $N$. Since $F_{K}$ is symmetric with respect to the rotation $w \rightarrow \exp (2 \pi i / \nu) w$, there are $N / \nu$ $F_{\Gamma}$ 's in the region $0 \leqq \arg w<2 \pi / \nu$. Using this fact, for example, $(3,3,11)$ does not exist. If such a signature existed, the order of the automorphism group would be 33 . Three $(=33 / 11)$ fundamental regions of a Fuchsian group with signature $(3,3,11)$ do not form one eleventh part of the fundamental region of any Fuchsian group since the angle at a vertex of a fundamental region must be $2 \pi / m$, where $m$ is an integer. In the same way, we find that $(2,5,10)$, 
$(3,3,11),(3,3,15),(3,5,5)$ and $(5,5,5)$ do not exist. Next, we show that $(5,5,15)$ and $(2,2,4,12)$ do not exist. If these signatures existed, the automorphism group would be cyclic. But these signatures do not satisfy the $1 . \mathrm{c} . \mathrm{m}$. condition. Furthermore, $(2,3,12),(2,3,22)$ and $(3,4,12)$ do not exist. The surface having an automorphism of order 12 or 22 is conformally equivalent to the hyperelliptic surface defined by

$$
y^{2}=x^{12}-1 \text { or } y^{2}=x\left(x^{11}-1\right),
$$

respectively, on which 12 Weierstrass points exist. If $p \in S$ is a Weierstrass point, every point in $G$-orbit of $p$ is also a Weierstrass point. Then for each signature $(2,3,12),(2,3,22)$ and $(3,4,12)$ the number of Weierstrass points should be represented as $96 m+48 \varepsilon_{1}+32 \varepsilon_{2}+8 \varepsilon_{3}, 66 m+33 \varepsilon_{1}+22 \varepsilon_{2}+3 \varepsilon_{3}$, and $24 m+$ $8 \varepsilon_{1}+6 \varepsilon_{2}+2 \varepsilon_{3}$, respectively, where $m$ is an integer and $\varepsilon_{j}=0$ or $1(j=1,2,3)$. But 12 cannot be represented in these ways. Then $(2,3,12),(2,3,22)$ and $(3,4,12)$ do not exist.

Finally we show the non-existence of $(2,5,6)$. Suppose that the group of order 60 with signature $(2,5,6)$ exists. 10 branch points of multiplicity 6 is regarded as branch points of multiplicity 3. The signature of the cyclic group generated by an automorphism of order 3 is $(0 ; 3,3,3,3,3,3,3)$ or $(1 ; 3,3,3,3)$. If the signature were the former, the surface would be conformally equivalent to the surface defined by

$$
y^{3}=\left(x-\alpha_{1}\right)^{2}\left(x-\alpha_{2}\right)^{2}\left(x-\alpha_{3}\right) \cdots\left(x-\alpha_{7}\right),
$$

where $\alpha_{1}, \cdots, \alpha_{7}$ are distinct complex numbers. But the Weierstrass gap sequence at $\left(\alpha_{1}, 0\right)$ and at $\left(\alpha_{3}, 0\right)$ are different. This contradicts that 10 branch points are equivalent under the group. Therefore, the signature of the cyclic group of order 3 must be $(1 ; 3,3,3,3)$. If two automorphism groups $\left\langle h_{1}\right\rangle$ and $\left\langle h_{2}\right\rangle$ of order 3 have a common fixed point then $\left\langle h_{1}\right\rangle=\left\langle h_{2}\right\rangle$. So the branch points of multiplicity 3 are divided into equivalence classes, and each class consists of 4 points. But 10 is not divisible by 4 . Thus, $(2,5,6)$ does not exist.

By virture of the existence of the group of order 64 with signature $(2,4,8)$, for $k \equiv 0(\bmod 8), N(5, k) \geqq 64$. And by virture of the existence of the group of order 48 with signature $(2,4,12)$, for $k \equiv 0,4(\bmod 12), N(5, k) \geqq 48$. So it is not necessary to consider the groups of order 48 with signatures $(2,6,6),(3,3,6)$, $(3,4,4)$ and $(2,2,2,3)$. Similarly, by virture of the existence of the signatures shown in $\S \S 4,5$, it is not necessary to consider the following signatures.

$\begin{array}{llll}48(2,6,6) & 48(3,3,6) & 48(3,4,4) & 48(2,2,2,3) \\ 32(4,4,4) & 32(2,2,2,4) & 24(3,6,6) & 24(2,2,2,6) \\ 20(2,2,2,10) & 16(2,2,4,4) & 16(2,2,2,2,2) & 16(1 ; 2) \\ 12(2,2,6,6) & 12(2,3,3,6) & 12(3,3,3,3) & 12(2,2,2,2,3) \\ 12(1 ; 3) & 11(11,11,11) & 10(2,2,10,10) & 10(2,2,2,2,5) \\ 10(1 ; 5) & & & \end{array}$


Summing up, we obtain our theorem.

\section{REFERENCES}

[1] HaRvey, W.J., Cyclic groups of automorphisms of a compact Riemann surface. Quart. J. Math. Oxford (2), 17 (1966), 86-97.

[2] Heins, M., On the number of 1-1 directly conformal maps which a multiplyconnected plane region of finite connectivity $p(>2)$ admits onto itself. Bull. Amer. Math. Soc. 52 (1946), 454-457.

[3] Kato, T., On the number of automorphisms of a compact bordered Riemann surface. Kōdai Math. Sem. Rep. 24 (1972), 224-233.

[4] Kato, T., On the order of automorphism group of a compact bordered Riemann surface of genus four. Kōdai Math. J. 7 (1984), 120-132.

[5] Macbeath, A.M., Discontinuous groups and birational transformations. Proc. Dundee Summer School, (1961).

[6] NAKAGAWA, K., On the orders of automorphisms of a closed Riemann surface. Pasific J. Math. 115, No. 2 (1984), 435-443.

[7] OiKaWA, K., Note on conformal mappings of a Riemann surface onto itself. Kōdai Math. Sem. Rep. 8 (1956), 23-30, 115-116.

[8] Tsuji, R., On conformal mappings of a hyperelliptic Riemann surface onto itself. Kōdai Math. Sem. Rep. 10 (1958), 127-136.

[9] TsuJI, R., Conformal automorphisms of a compact bordered Riemann surface of genus 3. Kōdai Math. Sem. Rep. 27 (1976), 271-290.

[10] Wiman, A., Über die hyperelliptischen Curven und diejenigen vom Geschlechte $p=3$ welche eindeutigen Transformationen in sich zulassen. Bihang Till. Kongl. Svenska Veienskaps-Akademiens Hadlinger 21 (1895-96), 1-23.

[11] Wiman, A., Über die algebraischen Curven von den Geschlechten $p=4,5$ und 6 welche eindeutige Transformationen in sich besitzen. Bihang Till. Kongl. Svenska Veienskaps-Akademiens Hadlinger 21 (1895-96), afd 1, no. 3, 41 pp.

NTT, Electrical Communication Laboratries 